Weckx, S.; D'Hulst, R.; Claessens, B.; Driesen, J., "Multiagent Charging of Electric Vehicles Respecting Distribution Transformer Loading and Voltage Limits," Smart Grid, IEEE

Transactions on , vol.PP, no.99, pp.1,1

Digital Object Identifier: 10.1109/TSG.2014.2345886

URL: http://ieeexplore.ieee.org/stamp/stamp.jsp?tp=\&arnumber=6881739

IEEE. Personal use of this material is permitted. Permission from IEEE must be obtained for all other users, including reprinting/ republishing this material for advertising or promotional purposes, creating new collective works for resale or redistribution to servers or lists, or reuse of any copyrighted components of this work in other works. 


\title{
Multi-agent charging of electric vehicles respecting distribution transformer loading and voltage limits
}

\author{
Sam Weckx, Student Member, IEEE, Reinhilde D'Hulst, Bert Claessens, Johan Driesen, Member, IEEE
}

\begin{abstract}
In this work a market based multi-agent control mechanism, that incorporates distribution transformer and voltage constraints for the charging of a fleet of EVs, is presented. The algorithm solves a utility maximization problem in a distributed way, assigning most charging power to the EVs with the highest need for energy. The algorithm does not rely on an iterative exchange of messages, but finds the optimal solution after the exchange of just one single message. A substation agent is responsible for guaranteeing a safe network operation. It uses the remaining capacity of each of the $\mathrm{EV}$ chargers for reactive voltage control. The performance of the algorithm is evaluated on an existing three-phase four-wire distribution grid. Simulation results show that the fleet of EVs can be charged at a minimal increase of costs, without jeopardizing the network.
\end{abstract}

Index Terms-Distributed optimization, distribution network, electric vehicle, multi-agent system, power curtailment, reactive power control, voltage control

\section{INTRODUCTION}

$\mathbf{T}$ HE number of electric vehicles $(\mathrm{EV} s)$ is expected to rise significantly in the future. A high penetration of EVs forms both a threat and an opportunity for the operation of the electricity network. EVs have high energy requirements and therefore are a considerable extra load for the distribution network. This can result in severe voltage drops or overloading of the distribution transformer. On the other hand, EVs are parked longer than required to charge completely, resulting in a significant amount of flexible consumption if the charging power of EVs can be controlled. Therefore EVs are interesting for the application of Demand Side Management (DSM) where the charging of EVs is shifted to reduce generation costs.

In case of a high excess of wind energy or the availability of cheap electricity all available EVs will preferably charge at maximum power. This might overload the low voltage network distribution transformer or make it difficult to comply with national standards for keeping the voltage within acceptable limits. The preferable implementation of DSM is able to combine both objectives by reducing generation costs, without jeopardizing the grid. In case the charging commands by an EV aggregator result in local grid problems, the system operators should have the option to intervene in demand response signals to ensure security of supply and quality of service.

As DSM will eventually involve millions of customers, centralized control will not be manageable because limits of

S. Weckx and J. Driesen are with the Department of Electrical Engineering ESAT-ELECTA, KU Leuven, Kasteelpark Arenberg 10, bus 2445, 3001 Leuven, Belgium. S. Weckx has a PhD fellowship of the Research Foundation - Flanders (FWO). R. D’Hulst and B. Claessens are with VITO, Mol, Belgium.

KULeuven and VITO are founding members of EnergyVille.

Corresponding author's email address: sam.weckx@esat.kuleuven.be computational complexity and communication overhead will be reached [1]. Different authors therefore propose multi-agent systems to obtain a scalable system. Algorithms based on dual decomposition and the alternating direction of multipliers (ADMM) are characterised by the iterative exchange of signals to obtain the optimal charging pattern for all EVs. These typically do not take network constraints into account [2]-[4]. In [5] ADMM is applied for the control of loads to minimize the losses in unbalanced distribution grids while taking into account network constraints. The total EV owners convenience is maximized for a single period without violating branch constraints by means of ADMM in [6]. The required iterative form of communication in these control algorithms might hinder a practical implementation and therefore is avoided in this work.

Many other multi-agent systems were proposed to coordinate the charging of EVs in a scalable way. In [7] a multiagent system is applied to maximize the load factor by the iterative exchange of price signals. Reducing peak demand is also obtained with decentralized control in [8]. A multiagent based Virtual Power Plant consisting of domestic devices was created in [9] to compensate imbalance caused by wind energy. The extra voltage drop caused by the charging of EVs, which is one of the main concerns of the Distribution System Operator (DSO), is however not taken into account in these control systems. [10] takes into account voltage constraints, but requires an exhaustive search to find the combination of schedules that minimizes the cost of charging the EVs.

In this paper an existing scalable market control system for DSM [11]-[15] of EVs is analysed and extended. Many extensions to this control heuristic have already been developed [1], [16], [17], and practical test sites exists where the market based control is tested [17]. Our first contribution to this control framework is a mathematical proof that shows that this control heuristic is in fact a method to solve a distributed utility maximization problem. The underlying problem is identical to the one of [2], [18], but the solution method does not result on an iterative form of communication. Secondly local distribution network constraints in unbalanced three-phase four-wire radial grids are added to this framework. Only those agents that are responsible for LV networks with network problems need to be updated. Both transformer overloading and voltage problems can be avoided. Finally reactive power is added as a control variable. It is shown that special care is required for reactive voltage control in three-phase four-wire unbalanced radial distribution networks.

This paper is structured as follows: In Section II, the market based multi-agent control framework is introduced. This framework is proven to be a distributed implementation of a 
utility maximization problem in section III. In the second part of this section the distributed utility maximization problem is extended with some network constraints and reactive voltage control in unbalanced networks is discussed. In Section IV the framework is compared to other algorithms to solve the utility maximization problem in a distributed way. The test system is described in Section V. Finally Section VI discusses the obtained results.

\section{MARKeT BASED MULTi-AGENT CONTROL}

The proposed multi-agent control is based on [11]-[15]. This control heuristic is based on a tree structure of three types of agents: device agents, substation agents and an auctioneer agent. All controllable consumer devices are equipped with a device agent. In a first step each device agent defines a bid function where the customer assigns the price, which is limited by the interval $[0,1]$, to have a certain power consumption level.

In a second step the device agents discretize the bid function and send it to a substation agent. This substation agent sums up the bid functions of all the underlying devices in a low voltage network. The substation agents in turn send the bid function to a unique auctioneer agent. This significantly reduces the amount of communication required and makes the system scalable. Finally the auctioneer agent will define the equilibrium price as the intersection of the aggregated bid functions and the supply bid function. The supply bid function represents the prices that the producer or balancing party will accept for different generation levels. When the equilibrium price is defined, this value is sent back to all the device agents that will select their corresponding power level. Fig. 1 presents this information hierarchy.

In this paper extra intelligence is added to the substation agent. The substation agent will only add up powers for a certain possible equilibrium price if these will not result in violation of the transformer or voltage limits. Moreover, it will apply the offered flexibility to avoid voltage problems. In this work the focus is on EVs, however the framework can be extended to other types of devices.

In [1], [16], [19], [20] an extension is made to the auctioneer agent to include planning in the framework. Based on this planning, the auctioneer is able to define the supply bid function for the real-time control. In this work the method of [1], [16] is applied, with the purpose of minimizing the total charging cost given a known day-ahead price. The charging should not lead to problems in the low voltage network. Other objective functions can be applied. More details about the applied approach can be found in [1], [16].

\section{AN OPTIMIZATION PERSPECTIVE ON MARKET BASED CONTROL}

In this section it will be proven that the heuristic described in section II can be interpreted as a utility maximization problem. This utility maximization problem can be extended with distribution network constraints and be solved in a distributed way.

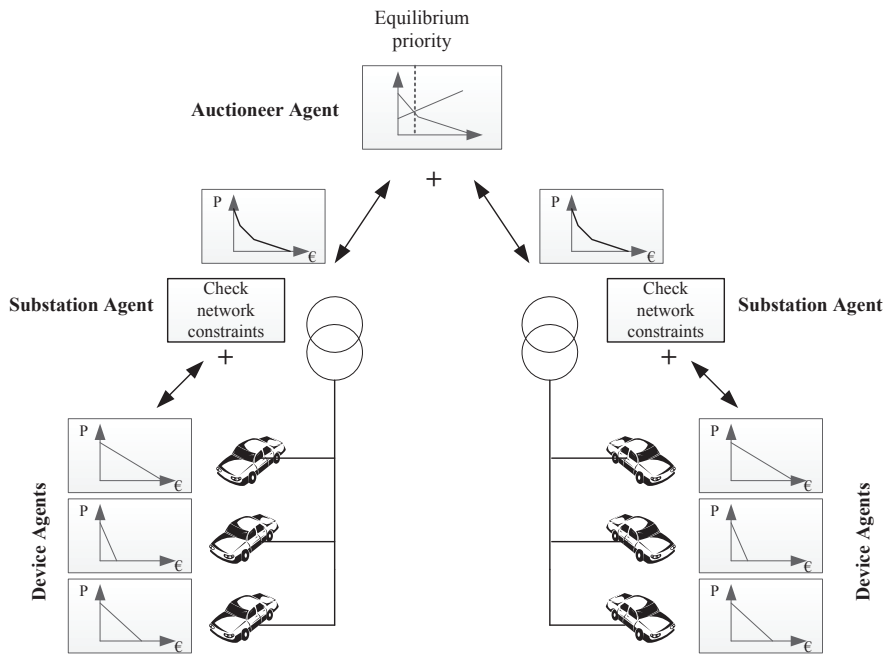

Fig. 1: Market based control of an electric network

\section{A. Utility function of an EV}

Depending on their state, energy may be worth a different value for each device. This is generally expressed by a utility function, a concept applied in microeconomics. In this paper is assumed that utility functions are decomposable in time. Popular decomposable utility functions $U\left(P_{i}\right)$ are the linear and quadratic utility function [18], where $P_{i}$ is the power consumption level for a specific time and $U\left(P_{i}\right)$ is the utility function of the EV $i$. An EV $i$ that consumes $P_{i} \mathrm{~kW}$ electricity at a rate of $\lambda$ per $\mathrm{kWh}$ is charged $\lambda P_{i}$ per hour. Hence, the welfare of each user is defined as [21]:

$$
W\left(P_{i}\right)=U\left(P_{i}\right)-\lambda P_{i}
$$

where $W\left(P_{i}\right)$ is the users welfare function. Given a certain price $\lambda$, the power $P_{i}^{*}(\lambda)$ that maximizes the welfare of $\mathrm{EV}$ $i$ is therefore defined as:

$$
P_{i}^{*}(\lambda)=\underset{P_{i}}{\operatorname{argmax}} U\left(P_{i}\right)-\lambda P_{i}
$$

The function defined by $P_{i}^{*}(\lambda)$ is the optimal bid a device would make to an auction based market. When this bid is used, each price will result in maximum welfare of the device. When the utility function is linear or quadratic, and the feasible sets are intervals, an explicit solution exists for $P_{i}^{*}(\lambda)$ [22]. The graphs shown at each EV in Fig. 1 are optimal bids that correspond to a quadratic utility function. A simple way of calculating a bid function for an EV is by using a corner price $p_{r}$ [23], [24], which is applied in this work. More on defining bid functions for EVs can be found in [1], [24]. In the heuristic described in section II the price $\lambda$ is not necessarily an actual price, but can be interpreted as a control signal.

The utility functions are not limited to linear or quadratic functions, but are assumed to be decomposable in time, nondecreasing and concave [21]. While the class of utility functions that fulfill these conditions is very large, it is convenient to have a quadratic utility function and the corresponding linear bid function [21], [25]. 


\section{B. Utility maximization problem}

In a utility maximization problem, the sum of all utilities of the customers, minus the cost $C(H)$ to deliver the total power $H$ is maximized [21]. This results in the following minimization problem:

$$
\begin{array}{ll}
\min _{P, H} & -\sum_{i=1}^{N} U\left(P_{i}\right)+C(H) \\
\text { subject to } & \sum_{i=1}^{N} P_{i}=H
\end{array}
$$

The constraint of this optimization problem ensures that the sum of the individual consumption of all EVs equals the total consumption of all EVs. The cost function $C(H)$ is assumed to be convex.

The total power $H^{*}(\lambda)$ that maximizes the welfare of the supplier is defined as:

$$
H^{*}(\lambda)=\underset{H}{\operatorname{argmax}} .-C(H)+\lambda H
$$

$H^{*}(\lambda)$ equals the supply bid function as presented in section II.

The dual of problem (3) is defined as:

$$
\max _{\lambda} \min _{P, H}-\sum_{i=1}^{N} U\left(P_{i}\right)+C(H)+\lambda\left(\sum_{i=1}^{N} P_{i}-H\right)
$$

where $\lambda$ is the Lagrange multiplier of the constraint (4). It will be shown in this section that the Lagrange multiplier equals the price charged to the customers in (1), (2). Therefore the same symbol is applied.

The Lagrangian dual function is decomposable in $P$ and $H$ and can be solved in a distributed way. The auction based heuristic is a distributed algorithm that solves the dual problem. If strong duality holds then the solution of the dual problem (6) equals the solution of the primal problem (3). It is supposed that there is a feasible solution and therefore Slaters condition holds [26]. This ensures a zero duality gap, and that optimal Lagrange multipliers exist.

The dual function can first be decomposed into subproblems that can be solved by each LV substation. It is assumed that there are $K$ substations. A substation agent is responsible for a subset of all the EVs, described by the set $n_{k}$. Each EV is part of one subset and the union of all subsets covers all EVs $N$. The subproblem of a LV substation $k$ in control of the subset of EVs $n_{k}$ is:

$$
\min _{P} . \quad-\sum_{i \in n_{k}} U\left(P_{i}\right)+\lambda \sum_{i \in n_{k}} P_{i}
$$

In the auction based heuristic a bid $P_{i}^{*}(\lambda)$ of a device $i$ corresponds to the solution of a subproblem of the dual function, as can be recognised in (2). The task of the substation agent is to add all the bids up to obtain $\sum_{i \in n_{k}} P_{i}^{*}(\lambda)$, which is the solution of subproblem (7) for each possible $\lambda$. Afterwards each substation agent sends the total consumption of their cluster for each possible equilibrium price to the auctioneer agent. The auctioneer adds up all these aggregated bids to obtain $\sum_{i=1}^{N} P_{i}^{*}(\lambda)$ and compares it to the power that the energy provider will produce given a certain price $H^{*}(\lambda)$ [21].

The market is cleared when the aggregated bids $\sum_{i=1}^{N} P_{i}^{*}(\lambda)$ equal $H^{*}(\lambda)$. It can be shown that $\sum_{i=1}^{N} P_{i}^{*}(\lambda)-H^{*}(\lambda)$ equals the subgradient of the dual problem [21], [27]. Therefore making the aggregated bids $\sum_{i=1}^{N} P_{i}^{*}$ equal to $H^{*}$ comes down to solving the dual problem (6). The Lagrange multiplier $\lambda$ of the dual problem is the equilibrium price, which is sent to the substation and EV agents.

\section{Utility maximization problem with transformer and voltage constraints}

The purpose of this paper is to include transformer and voltage limitations in the scalable heuristic. These network constraints can be included in the utility maximization formulation. Different constraints need to be added to the subproblem (7) of each LV substation. First of all transformer overloading in a LV substation $k$ has to be avoided. Therefore a constraint on the maximal apparent power is introduced:

$$
\left(P_{k}^{L}+\sum_{i \in n_{k}} P_{i}\right)^{2}+\left(Q_{k}^{L}+\sum_{i \in n_{k}} Q_{i}\right)^{2} \leq\left(S_{k}^{\max }\right)^{2}
$$

where $P_{k}^{L}$ and $Q_{k}^{L}$ are the uncontrollable active and reactive load flowing in the transformer of substation $k$ during the previous time step. $P_{k}^{L}$ and $Q_{k}^{L}$ can be obtained from a measurement of the total load flowing in the transformer, of which the load of the $n_{k}$ EVs is subtracted. The substation agents know the charging powers of all EVs during the previous time step. During the next time step, the sum of the uncontrollable load and the assigned charging power of the EVs may not trespass the maximal allowed apparent power $S_{k}^{\max }$

As the uncontrollable load might change slightly during the next time step and system losses will increase for high loads, it is recommended to include a small conservative margin on the maximal allowed apparent power $S_{k}^{\max }$. In a three-phase system, constraint (8) has to be formed for each separate phase.

A second issue that has to be taken into account is the significant voltage drop caused by the charging of EVs in LV networks. The influence of EVs on the voltage magnitude can be approximated with a linear model [28]-[31], resulting in an affine constraint. The voltage at a node $i$ connected to a substation $k$ can be approximated as:

$$
\left|V_{i}\right| \approx\left|V_{i}^{\text {base }}\right|+\sum_{m \in n_{k}}\left(\mu_{i, m}^{P} P_{m}+\mu_{i, m}^{Q} Q_{m}\right)
$$

where

- $\mu_{i, m}^{P}$ is the sensitivity of the voltage magnitude in node $i$ by active power injected at node $m$;

- $\mu_{i, m}^{Q}$ is the sensitivity of the voltage magnitude in node $i$ by reactive power injected at node $m$; 
- $V_{i}^{\text {base }}$ is the voltage at node $i$ due to the base load of the households;

- $V_{i}$ is the expected voltage at node $i$ with EVs charging; All the voltages should be limited between a minimum and maximum voltage:

$$
V^{\min } \leq\left|V_{i}\right| \leq V^{\max }
$$

where $V^{\text {min }}$ and $V^{\text {max }}$ are the minimum and maximum allowed voltage. Typically, the voltages of a limited amount of control points at the end of the feeders have to be considered, as these are subject to the largest voltage deviations. It is assumed that these voltages are measured and communicated to the substation agent. The substation agent knows at what rate the EVs were charging during the previous control time step. With the voltage sensitivity factors the substation agent can calculate the effect these EVs had on the voltage during that measurement, to obtain the voltage caused only by the base load $V_{i}^{\text {base }}$. This voltage by the base load $V_{i}^{\text {base }}$ can then be used to plan the consumption of the EVs during the next time step of the control framework.

In real-life conditions the base load will vary during the next time step of the control framework. Therefore the obtained $V_{i}^{\text {base }}$ is only an estimate of the voltage that will occur during the next time step by the base load only. This could result in small violations of constraint (10). Besides that there will be linearisation errors by the linear model. Therefore a small extra conservative margin is introduced in the limits of (10). In this work $V^{\min }$ and $V^{\max }$ will be chosen to be $\pm 9 \%$ of the nominal voltage, whereas the actual limits equal $\pm 10 \%$ of the nominal voltage.

An important advantage of the application of the linear voltage model is that the substation agent does not need to know the actual inelastic base load of the households to approximate the voltage caused by the base load only. The inelastic load profiles might contain privacy sensitive information. Another reason for the application of the linear model is that the constraints remain a convex set. This will reduce the computation time. The sensitivity factors can be based on off-line calculations that are seldom updated depending on the network operation, e.g., heavy or light loading conditions [32]. Another advantage is that these sensitivity factors can be approximated based on historic smart meter data, without having information about the exact grid topology [33].

For a single-phase line with mainly resistive line impedance, reactive power has a limited effect on the voltage [34], [35]. However special care is required in three-phase four-wire distribution grids. The connection of single-phase loads and EVs to three-phase four-wire LV networks will not only alter the voltage profile in the connected phase, but also the two other phase voltages due to a neutral point shift [36]. I.e. in grids with mainly resistive line impedance, reactive power absorption in phase $U$ will reduce the phase voltage of phase $\mathrm{U}$, while it significantly increases the phase voltage of phase $\mathrm{W}$ and decreases the phase voltage of phase V. The neutralpoint shift origins from the return current through the neutral conductor. This effect is exploited by the substation agent, as will be shown in section VI.
The remaining capacity of the EV charger can be applied for reactive voltage support in the network by the substation agent. When reactive voltage control is applied, customer utility can be increased for low equilibrium prices, as the reactive power injection partly compensates the voltage drop caused by the increased load. The EV charger complex output power is limited by:

$$
\left(P_{i}\right)^{2}+\left(Q_{i}\right)^{2} \leq\left(S_{i}^{\mathrm{Nom}}\right)^{2}
$$

Where

- $Q_{i}$ is the reactive power by the EV charger connected to node $i$;

- $S_{i}^{\text {Nom }}$ is the nominal apparent power of the EV charger connected to node $i$;

To add this constraint to the subproblem of the substation agent it is assumed that besides the bid function the EV also sends $\left|S_{i}^{\text {Nom }}\right|$ to the substation agent. If this is not communicated, the substation agent can replace $\left|S_{i}^{\text {Nom }}\right|$ with $P_{i}^{\max }$, which the substation agent can extract from the received bid function. A constraint on the power factor can be set by a linear constraint:

$$
Q_{i} \leq \gamma P_{i}
$$

where $\gamma$ is a constant defining the allowable ratio between reactive and active power. In this work the constraint on the power factor is omitted and it is assumed that the full nominal apparent power can be used for reactive power control if necessary. When reactive power is used, it is advised to add a small quadratic penalty term for reactive power to the objective function of the utility maximization problem, to avoid the use of reactive power when no voltage problems occur.

The utility maximization problem with transformer and voltage constraints becomes:

$$
\begin{array}{ll}
\min _{P, Q, H} & -\sum_{i=1}^{N} U\left(P_{i}\right)+C(H) \\
\text { subject to } & \sum_{i=1}^{N} P_{i}=H \\
& (8-10) \forall k=1 \ldots K \\
& (11-12) \forall i=1 \ldots N
\end{array}
$$

Constraints (8-10) have to hold for each of the $K \mathrm{LV}$ substations, making scalability even more important. Constraints (11-12) are only added when the substation agent also applies reactive power for grid voltage control. These constraints are specific for each EV, and therefore need to be handled in a scalable way. For the ease of simplicity the small quadratic penalty term for reactive power is not presented in the objective function of (13).

By relaxing the first constraint of (13), the following dual formulation is obtained:

$$
\begin{gathered}
\max _{\lambda} \min _{P, Q, H}-\sum_{i=1}^{N} U\left(P_{i}\right)+C(H)+\lambda\left(\sum_{i=1}^{N} P_{i}-H\right) \\
\text { subject to }(8-10)
\end{gathered}
$$$$
(11-12)
$$ 
The Lagrangian dual function is decomposable in $P$ and $H$ and can be solved in a distributed way. There will be a substation agent at each LV substation of the grid. Each of the substation agents will solve a subproblem of (14). The subproblem of a substation agent located at a LV substation $k$ equals:

$$
\begin{array}{ll}
\min _{P, Q} & -\sum_{i \in n_{k}} U\left(P_{i}\right)+\lambda \sum_{i \in n_{k}} P_{i} \\
\text { subject to } & (8-10) \\
& (11-12)
\end{array}
$$

Compared to the subproblem of (7), transformer and voltage constraints have been added. The substation agent receives the utility function of all EVs of its cluster and solves optimization problem (15) for each possible equilibrium price $\lambda$. In a practical implementation, possible equilibrium prices are limited between 0 and 1 . This interval is divided into 100 possible equilibrium prices $(\triangle \lambda=0.01)$. Optimization problem (15) will be solved for each of these 100 possible prices at the substation level. Gurobi is applied to solve this optimization problem [37]. The solution $\sum_{i \in n_{k}} P_{i}^{*}(\lambda)$ of subproblem (15) is sent to the auctioneer agent who can clear the market after adding up all of the solutions of the $K$ substation agents. It can again be shown that $\sum_{i=1}^{N} P_{i}^{*}(\lambda)-H^{*}(\lambda)$ equals the subgradient of the dual problem [27] and that making the aggregated bids $\sum_{i=1}^{N} P_{i}^{*}$ equal to $H^{*}$ comes down to solving the dual problem (14). Note that this solution complies with all network limitations in the LV substations for each equilibrium price. The outcome of the market clearing by the auctioneer agent results in an equilibrium price $\lambda^{*}$, which is sent to the substation agents. The substation agents can then send the active and reactive power setpoint to each EV.

One could argue that solving optimization problem (15) for each possible equilibrium price is very time consuming. However optimization problem (15) is convex and can therefore be solved very efficiently for each possible equilibrium price. The different optimization problems can easily be parallellized. To further reduce the amount of computations, it can first be checked for which prices the network constraints (8-10) will be violated. Only for those prices optimization problem (15) will have to be solved, whereas for the prices without network problems it suffices to just add the different bids as done to solve the problem without network constraints (7). The prices that cause network constraint problems can be found in one load flow. After this, optimization problem (15) is solved for all of the problematic prices. Further reductions in computation time can be obtained by approximating constraint (11) by a polygon [28].

The total procedure is presented in Fig. 2. After the devices have sent their utility function, the substation agents can start maximizing total consumer welfare for all possible equilibrium prices. They start with an initial small price and keep on incrementing the possible equilibrium price until the maximum price is reached. Results for both active and reactive power are

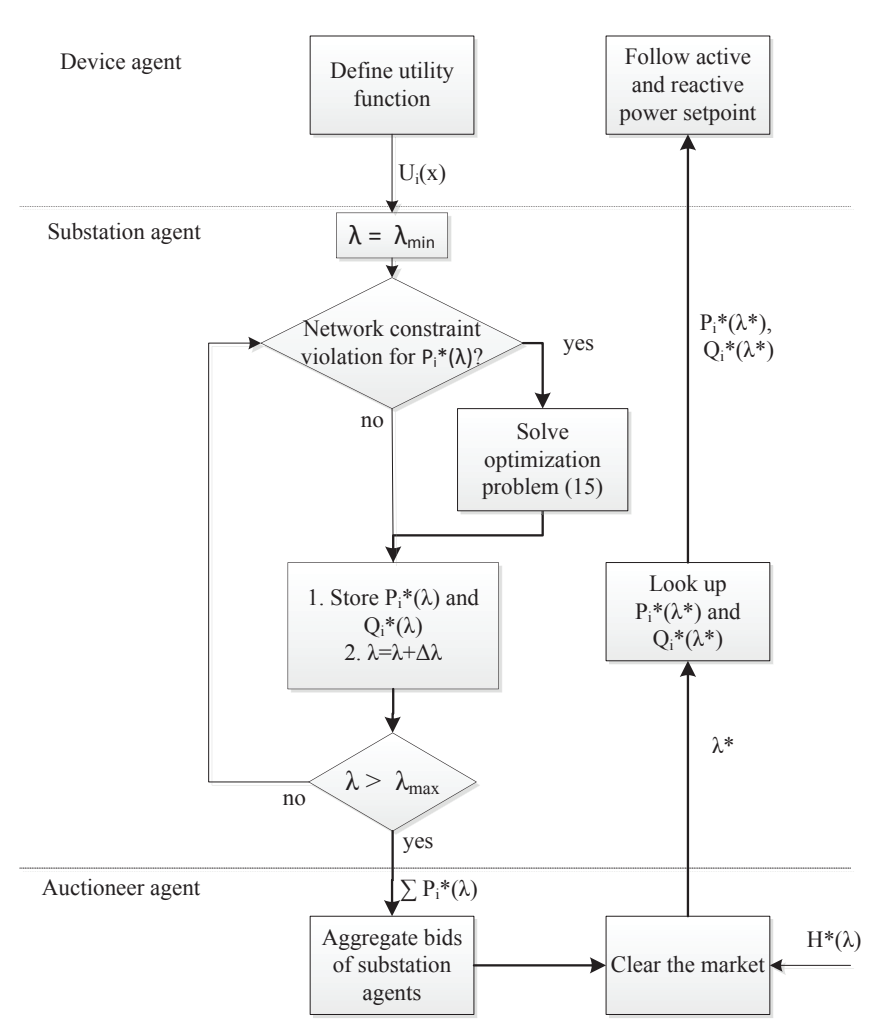

Fig. 2: Flowchart of the distributed utility maximization that incorporates network constraints.

stored by the substation agents. When the power consumption levels are defined for all the possible equilibrium prices, the aggregated bid function is sent to the auctioneer agent. This agent can then define the final equilibrium price $\lambda^{*}$. Once this final price is received by the substations, the substation agents can look up the power setpoints and send them to all EVs. For the ease of presentation, the substation agent calculations are presented as a loop, however the substation agent can also compute the aggregated bids in parallel.

One of the advantages of the suggested approach is that neither the device agents nor the auctioneer agents have to change compared to the existing market based control of section II. All intelligence is added to the substation agents, who are the only agents that have to be aware of the grid conditions. It is possible to equip only those LV networks that are sensitive to network problems with the substation agent that takes into account the network limitations or to gradually introduce the new substation agent in the market based control of section II.

\section{COMPARISON WITH OTHER DISTRIBUTED UTILITY MAXIMIZATION ALGORITHMS}

Distributed utility maximization algorithms in smart grid literature are mainly based on dual ascent methods [2], [21], [22], [38]-[40] to solve the dual problem (14). In a dual ascent method an iterative exchange of messages is applied to find the optimal solution of the dual problem. Each iteration the central agent broadcasts a price. The device agents send back 
their planned consumption for this price. After this iteration, the price is updated by the central agent and a new iteration starts. These iterations continue until convergence of the price takes place.

Both dual ascent methods as well as the proposed heuristic can be used to solve the exact same problem, but they differ in their communication and computational requirements. The proposed method has some advantages and some disadvantages compared to these dual ascent methods.

\section{A. Comparison of communication requirements}

The proposed heuristic has mainly communicational benefits compared to the dual ascent methods. First of all it is not based on an iterative communication procedure in contrast to dual ascent methods. The device agents only communicate their utility function to the substation agents once. The utility functions can be compressed for an efficient communication, i.e. quadratic utility functions can be represented by three constants. The substation agents aggregate the bids and deliver these to the auctioneer agent. When the auctioneer agent has received all of the aggregated bids, he can immediately define the Lagrange multiplier of the dual problem (6), resulting in convergence in a single iteration. Only one price is sent back by the central agent. In a dual ascent implementation, the central agent needs to communicate a possible equilibrium price to all of the device agents every iteration. Therefore the amount of communication from the central agent to the device agents is significantly higher. Each iteration, the device agents need to respond to the higher agents when dual ascent methods are applied. So the amount of communication from the device agents to the substation agent can be expected to be higher. Besides that, iterative algorithms will have a higher overhead, i.e. a connection needs to be established at each iteration to send a packet. Moreover, in a realistic communication environment the underlying infrastructure places constraints on the communication, such as delays or maximum throughput [16]. The heuristic can also be made event-based to reduce the amount of communication [16]. Just as in the dual ascent methods, the utility function of each consumer remains unknown for the central agent due to the aggregation of the individual utility functions at substation level, ensuring privacy.

\section{B. Comparison of computational requirements}

A clear disadvantage of the proposed method is that the substation agent also solves (15) for values of $\lambda$ that are far from optimal, whereas dual ascent methods converge towards the optimal $\lambda$. If the optimal $\lambda$ always evolves smoothly in time, these less efficient computations can be avoided by only solving the required problem (15) in the neighbourhood of the previous optimal $\lambda$.

Another disadvantage compared to the dual ascent methods is the requirement that utility functions have to be decomposable in time. The utility functions should reflect who has the highest need for the available energy at the actual time step, independent of future information. To counteract this disadvantage, a planning approach is developed in [1], [16],
[19], [20] to optimize the supply bid function at each time step.

An advantage of the proposed heuristic is that it can work with utility functions that are just convex and not necessarily strict convex. It can even handle binary behaving devices [20]. The local subproblem of the substation agent (15) then becomes an integer problem, which can be efficiently solved locally at the substation agent. Dual ascent methods can not guarantee convergence for this type of problem [41]. Dual ascent methods also require special care for a good step-size selection. Another benefit of the proposed heuristic is that the local intelligence at device level is minimal. There is no need to solve an optimization problem at device level, in contrast to many dual ascent methods. This can reduce hardware and software requirements at device level.

\section{Simulated NETWORK}

The network used in the simulations is an existing threephase four-wire radial distribution system with a TT earthing arrangement in Belgium. The network consists of 62 customers and is depicted in Fig. 3. This network is a semi-urban reference network in the LINEAR project [42].

All main feeder cables are of type EAXVB $1 \mathrm{kV}$ $4 \times 150 \mathrm{~mm}^{2}$ except for the cable between the substation and node 1 , which is of type EAXVB $1 \mathrm{kV} 4 \times 95 \mathrm{~mm}^{2}$. Cable properties are defined in table I. The impedance values are calculated according to design specifications in the Belgian standard for underground distribution cables NBN C33-322 [43] with an assumed operating temperature of $45^{\circ} \mathrm{C}$. All households have a single-phase connection with a nominal line-to-neutral voltage of $230 \mathrm{~V}$ and are equally spread across the three phases. Statistically representative residential load profiles are available to perform load flow simulations. A constant power load model was assumed. Generation of these load profiles is described in [44]. No data was available on reactive power consumption by the loads which is therefore neglected in the simulation.

The time step used in the simulation is one minute. EVs are connected by single-phase connections to the network with a maximum capacity of $3.3 \mathrm{~kW}$. Each household is assumed to possess an EV. The EV charging energy and the arrival and departure time are modelled based on Flemish data on travel behavior as described in [45]. It is assumed that they will only charge at home.

The 10 minutes mean r.m.s. voltage is limited to $\pm 10 \%$ of the nominal voltage. According to Belgian regulation, the DSO has to comply with the power quality standard EN-50160. The voltage control by the substation agent is executed every 15 minutes in this work. Therefore it is possible that due to a sudden increase of the base load the voltage drops further below the acceptable limits as set by the standard, and the substation agent cannot respond fast enough to keep the 10 minutes mean r.m.s. voltages within the required range of \pm $10 \%$ of the nominal voltage. This is a rare event and the 10 minutes mean r.m.s. voltage remains within the range of \pm $10 \%$ of the nominal voltage, during more than $95 \%$ of the time as will be shown in section VI. 


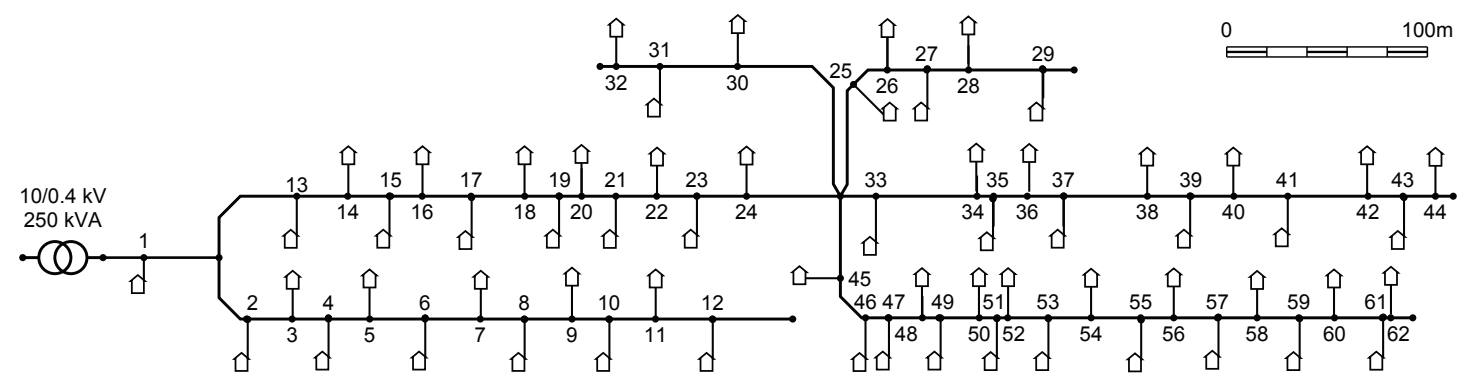

Fig. 3: The network used in the simulations. All lengths are drawn to scale.

TABLE I: Properties of the simulated network

\begin{tabular}{l|r|l} 
Properties & Value & Unit \\
\hline \hline Total feeder length & 1657 & {$[\mathrm{~m}]$} \\
Impedance of EAXVB $1 \mathrm{kV} 4 \times 95 \mathrm{~mm}^{2}$ & $0.352+0.078 \mathrm{j}$ & {$[\Omega / \mathrm{km}]$} \\
Impedance of EAXVB $1 \mathrm{kV} 4 \times 150 \mathrm{~mm}^{2}$ & $0.227+0.078 \mathrm{j}$ & {$[\Omega / \mathrm{km}]$}
\end{tabular}

\section{RESULTS}

In a first simulation the focus is on a single time step at 8 $\mathrm{pm}$. The aggregated bid function of only one substation agent will be investigated, to give a clear presentation of the results. The different grid conditions that will occur as a function of each possible equilibrium price are shown in Fig. 4. In Fig. 4a the aggregated bid function of the substation agent $\sum_{i \in n_{k}} P_{i}^{*}(\lambda)$ is plotted as a function of the equilibrium price. For equilibrium prices close to zero, an extra consumption of more than $160 \mathrm{~kW}$ can be activated. However, the distribution grid cannot host this extra consumption. From a certain equilibrium price, voltage drops caused by the charging of EVs become severe as presented in Fig. 4b. It is shown that starting from a price of $\lambda=0.25$, the voltage in phase $\mathrm{V}$ according to the linear model of (9) is expected to reach the minimum allowed voltage of 0.91 p.u. To be able to still increase the consumption in phase $\mathrm{V}$, reactive power will be injected in phase $\mathrm{V}$ and $\mathrm{U}$, and absorbed in phase $\mathrm{W}$ as shown in Fig. 4c. Due to the neutral point shift, reactive power absorption in phase $\mathrm{W}$ can boost the voltage in phase V. For lower equilibrium prices, the consumption in phase $\mathrm{W}$ increases, and the absorption of reactive power in this phase needs to be reduced. Starting from a price of $\lambda=0.05$ the aggregated bid function will be lower than for the case without network control, because the available reactive power is insufficient to compensate for the voltage drop caused by the charging of the EVs. In Fig. $4 \mathrm{~d}$ the loading of each phase of the transformer is depicted. The transformer capacity is high enough, so no transformer overloading takes place. The loading with and without the active and reactive power control is similar.

In a second test case, the same time step is investigated, but the maximum capacity of the transformer is cut in half to $125 \mathrm{kVA}$. Obviously an extra consumption of $160 \mathrm{~kW}$ would damage the transformer. From a certain equilibrium price the sum of the inelastic load and the extra consumption by EVs reaches the maximum capacity of the transformer. No more extra consumption can be activated for lower equilibrium prices. For this case there is almost no benefit of reactive power control, as it only causes an extra loading of the distribution transformer. This is shown in Fig. 5. For equilibrium prices lower than approximately 0.25 , each of the phases would be loaded with more than the allowed 125/3 kVA. The available reactive power to solve voltage issues is therefore strictly limited. The total power consumption of the cluster of loads is now limited to approximately $100 \mathrm{~kW}$.

In the next simulation a time period of three days is investigated. A vehicle fleet needs to be charged as cheap as possible, given the day-ahead price. A day-ahead price of the Belgian spot market is used. During night electricity prices are lower, which is therefore the preferred time period for charging all the EVs. At the time period of the lowest price, all available cars preferably charge at maximum power. Even with a low base load, this will result in a severe voltage drop. The supply bid function in each time step is defined by the method developed in [1], [16]. Fig. 6 plots the power consumed during three days and the minimum 10 minute mean r.m.s voltage in the grid. All voltages are obtained by applying the backwardforward sweep power flow method [46]. To clearly present the algorithm, the data of only one substation is plotted. The high peak due to the simultaneous charging of EVs results in severe voltage drops. When the substation agent does take the network constraints into account, the power consumed during the lowest price period slightly drops, but the voltage remains within acceptable limits. If the substation agent can use the remaining capacity of the EV chargers for reactive power support, more EVs can charge simultaneously before voltage limits are reached. The reduced maximal charging power during the lowest price period results in a small price increase to charge the EV fleet. In comparison to the situation where network limitations are not taken into account, the total charging cost increases with $0.4 \%$ when active and reactive power are applied to comply with the network limits and $0.8 \%$ when only active power is used.

The same simulation can be performed for transformers with a transformer capacity of only $125 \mathrm{kVA}$. The peaks due to simultaneous charging of the EVs are now limited, to ensure that the transformer does not get overloaded as shown in Fig. 7. The transformer loading is plotted instead of the minimum grid voltage, because it is the transformer capacity that limits the total consumption by the EVs in the substation. Due to the strict limitations of the transformer, reactive power control is rarely used by the substation agent. The maximum transformer 


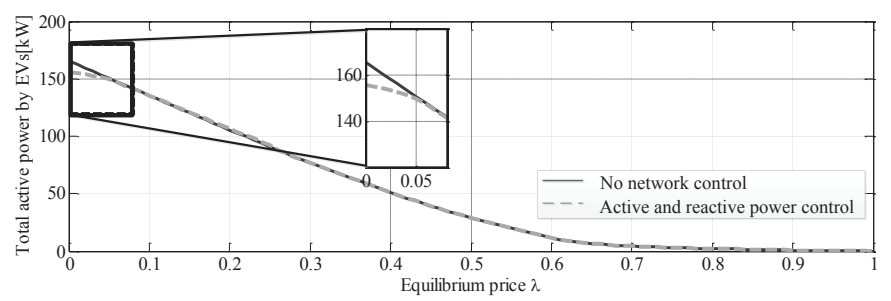

(a) The total consumption by the cluster as a function of the equilibrium price.

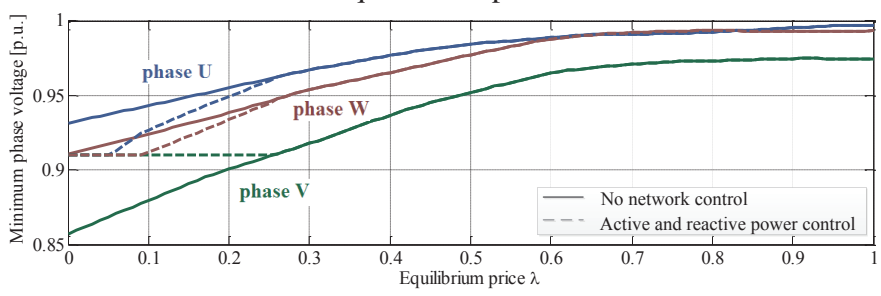

(b) The expected minimum phase voltages according to the linear model as a function of the equilibrium price.

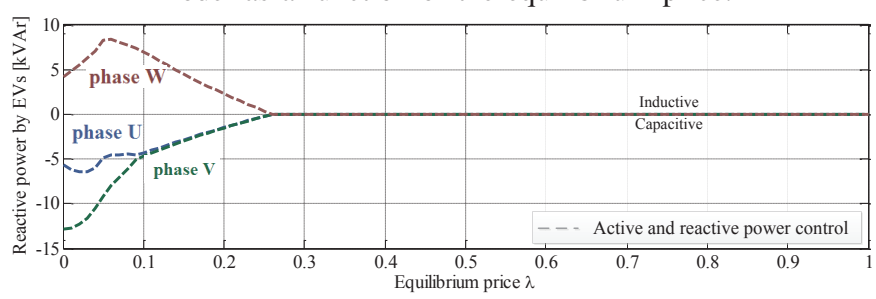

(c) The required reactive power by the EVs as a function of the equilibrium price.

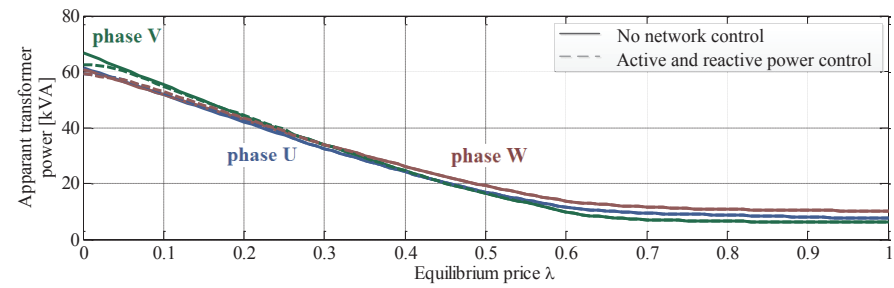

(d) The expected transformer loading as a function of the equilibrium price.

Fig. 4: Expected grid conditions as a function of the equilibrium price for a transformer with a capacity of 250

kVA.

loading in each phase is now $125 / 3 \mathrm{kVA}$. Load variations between two market clearings can result in a short violation of the transformer limits. Due to the lower consumption possible during the lowest price period, the total charging cost increases by $6 \%$ for both control approaches.

In Fig. 8 one week is simulated and the corresponding cumulative distribution function of the minimum voltage occurring in the grid is plotted. The voltage clearly complies with the power quality standard EN-50160.

In a final simulation the computational scalability is investigated. Fig. 9 presents the computation time as a function of the number of substations. Each of these substations has the topology of Fig. 3, with cable lengths that vary $1 \%$. In the multiagent algorithm, each substation agent needs to calculate the aggregated bid function before the auctioneer agent can clear

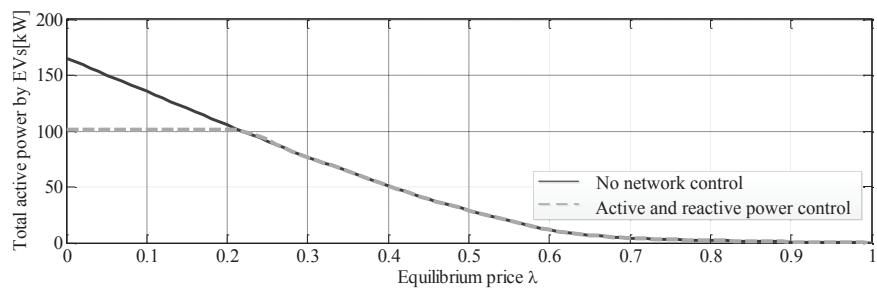

(a) The total consumption by the cluster as a function of the equilibrium price.

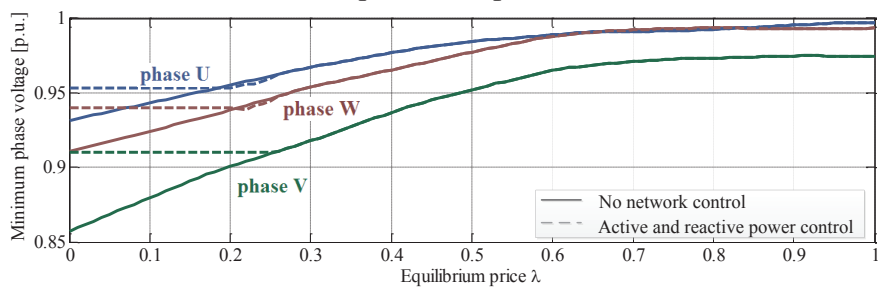

(b) The expected minimum phase voltages according to the linear model as a function of the equilibrium price.

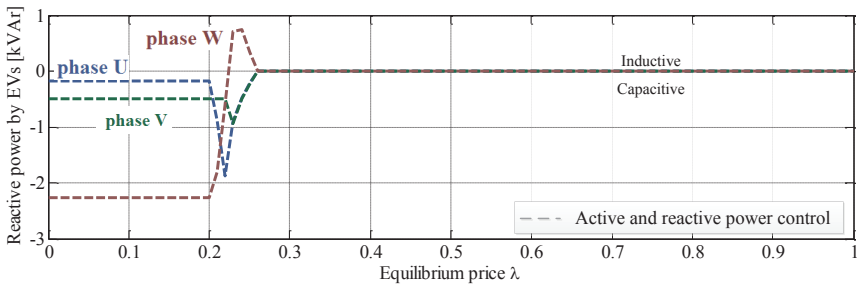

(c) The required reactive power by the EVs as a function of the equilibrium price.

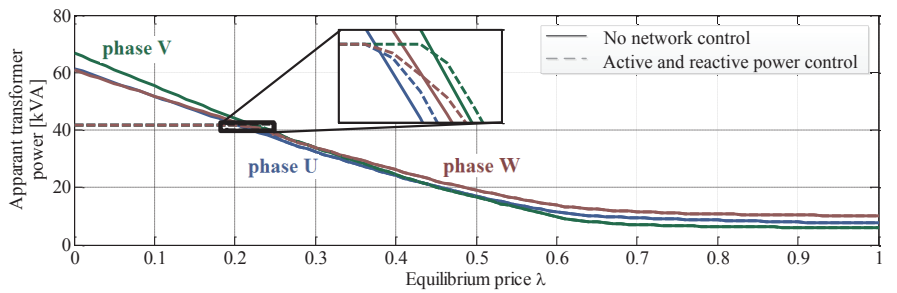

(d) The expected transformer loading as a function of the equilibrium price.

Fig. 5: Expected grid conditions as a function of the equilibrium price for a transformer with a capacity of 125

kVA.

the market. The slowest of all agents defines the computation time seen in Fig. 9. The addition of a slower agent therefore explains the increase in steps of the computation time. The execution time of a centralized algorithm keeps on increasing for the number of substations. The simulations were performed on a workstation using a Intel Xeon $(2.4 \mathrm{GHz}, 12 \mathrm{MB}$ cache, 4 cores) and $32 \mathrm{~GB}$ of RAM. A detailed discussion of the scalability of the original framework described in Section II can be found in [1].

\section{CONCLUSION}

In this work, a multi-agent market based control is proposed that takes into account transformer and voltage limitations. The control framework solves a utility maximization problem with LV network constraints. With the mutual exchange of only one 

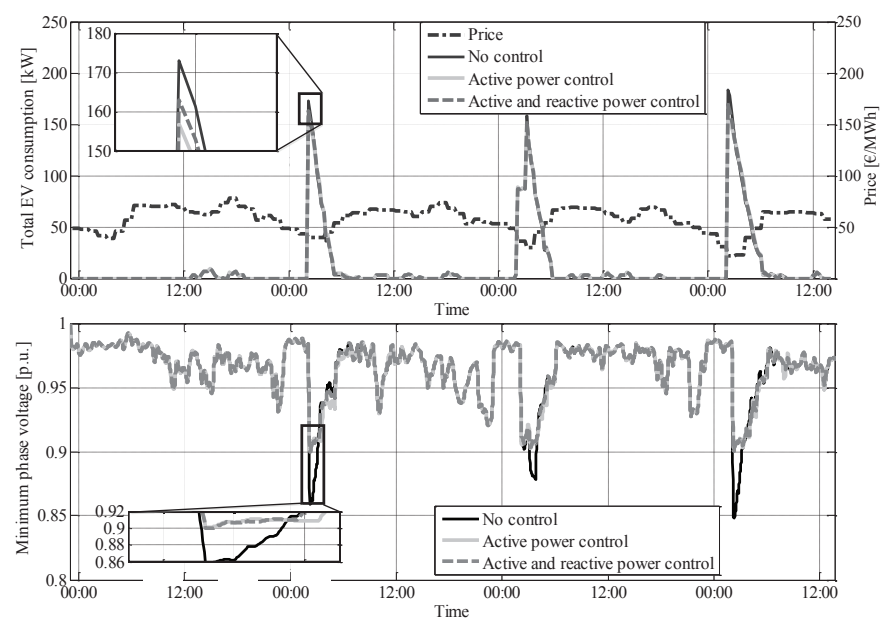

Fig. 6: Power consumption of the fleet of EVs (Top) and the minimum voltage in the grid (Down).
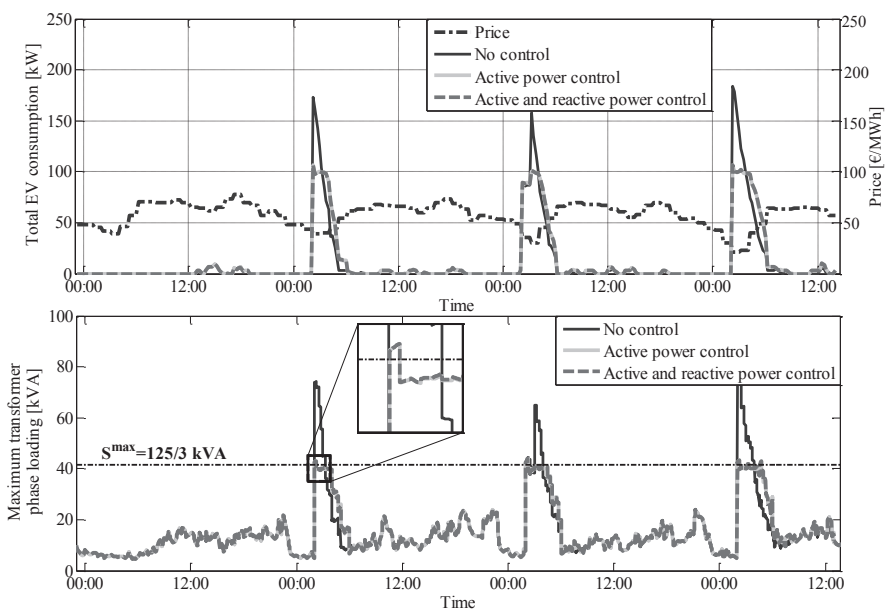

Fig. 7: Power consumption of the fleet of EVs (Top) and the maximum transformer loading for a transformer with a reduced transformer capacity of $125 \mathrm{kVA}$ (Down).

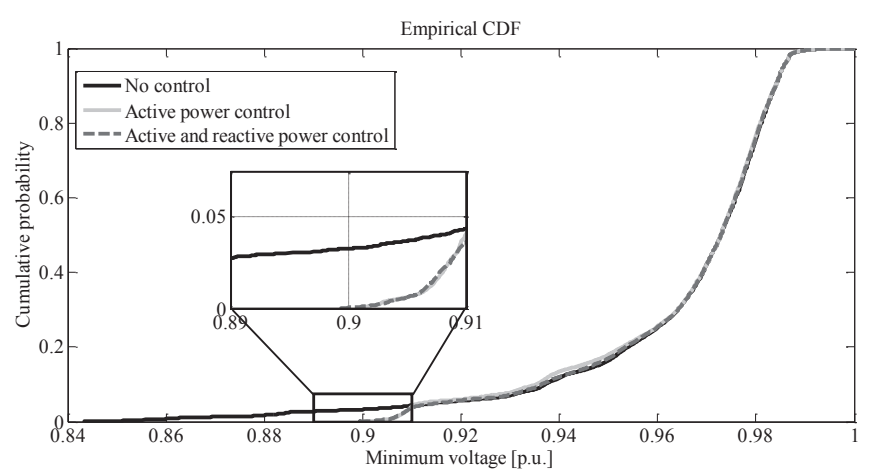

Fig. 8: Cumulative distribution function of the minimum voltage.

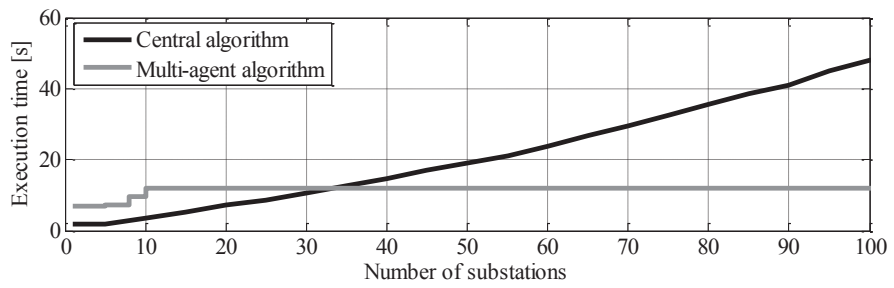

Fig. 9: Execution time for an increasing amount of substations.

message between the different agents, the utility maximization problem is solved. It is however required that the applied utility functions are decomposable in time.

Simulations show that with the proposed structure the fleet of EVs can be charged at minimum costs, without harming the network and while customer welfare remains maximal at all times. The remaining capacity of the EVs is used to provide reactive voltage support. Due to the application of reactive voltage control, more EVs can simultaneously charge before the voltage reaches critical limits. Therefore more EVs can charge at the same time during periods of low energy costs. Special attention has been given to the neutral point shift effect when applying reactive voltage control in unbalanced three-phase four-wire LV grids. Reactive voltage control has no benefits if the transformer capacity limits the amount of EVs that can charge simultaneously.

The focus of further and ongoing work is on an event-based implementation as in [16], rather than having fixed moments for the communication between the agents. Interaction between the agents could be made event-driven, like the arrival of a new EV or the violation of a voltage limit due to a sudden increase in household loads. This way, the substation agent could react faster to conditions that might harm the LV grid. This can have implications on the preferred communication and metering infrastructure. Besides that, it will be investigated how to incorporate utility functions that are not decomposable in time. Finally other business cases could be evaluated, like absorbing an unexpected excesses of wind power with the EV fleet.

\section{REFERENCES}

[1] S. Vandael, B. Claessens, M. Hommelberg, T. Holvoet, and G. Deconinck, "A scalable three-step approach for demand side management of plug-in hybrid vehicles," IEEE Trans. Smart Grid, vol. 4, no. 2, pp. 720-728, 2013.

[2] Z. Fan, "A distributed demand response algorithm and its application to phev charging in smart grids," IEEE Trans. Smart Grid, vol. 3, no. 3, pp. 1280-1290, Sept 2012.

[3] L. Gan, U. Topcu, and S. Low, "Optimal decentralized protocol for electric vehicle charging," IEEE Trans. Power Syst., vol. 28, no. 2, pp. 940-951, 2013.

[4] Z. Ma, D. S. Callaway, and I. A. Hiskens, "Decentralized charging control of large populations of plug-in electric vehicles," IEEE Trans. Control Syst. Technol., vol. 21, no. 99, pp. 67 - 78, 2013.

[5] E. DallAnese, H. Zhu, and G. Giannakis, "Distributed optimal power flow for smart microgrids," IEEE Trans. Smart Grid, to be published.

[6] C.-K. Wen, J.-C. Chen, J.-H. Teng, and P. Ting, "Decentralized plug-in electric vehicle charging selection algorithm in power systems," IEEE Trans. Smart Grid, vol. 3, no. 4, pp. 1779-1789, 2012.

[7] E. Karfopoulos and N. Hatziargyriou, "A multi-agent system for controlled charging of a large population of electric vehicles," IEEE Trans. Power Syst., vol. 28, no. 2, pp. 1196-1204, May 2013. 
[8] S. D. Ramchurn, P. Vytelingum, A. Rogers, and N. Jennings, "Agentbased control for decentralised demand side management in the smart grid," in The 10th Int. Conf. on Autonomous Agents and Multiagent Systems - Volume 1, ser. AAMAS '11, Richland, SC, 2011, pp. 5-12.

[9] B. Roossien, A. van den Noort, R. Kamphuis, F. Bliek, M. Eijgelaar, and J. de Wit, "Balancing wind power fluctuations with a domestic virtual power plant in europe's first smart grid," in Proc. IEEE Trondheim PowerTech, 2011, pp. 1-5.

[10] P. Papadopoulos, N. Jenkins, L. Cipcigan, I. Grau, and E. Zabala, "Coordination of the charging of electric vehicles using a multi-agent system," IEEE Trans. Smart Grid, vol. 4, no. 4, pp. 1802-1809, Dec 2013.

[11] K. Kok, C. Warmer, and I. Kamphuis, "Powermatcher: multiagent control in the electricity infrastructure," in Proc. of the 4th Int. joint Conf. on Autonomous agents and multiagent systems. ACM, 2005, pp. 75-82.

[12] K. Kok, C. Warmer, and R. Kamphuis, "The powermatcher: Multiagent control of electricity demand and supply," IEEE Intell. Syst., vol. 21, no. 2, pp. 89-90, 2006.

[13] K. Kok, B. Roossien, P. MacDougall, O. van Pruissen, G. Venekamp, R. Kamphuis, J. Laarakkers, and C. Warmer, "Dynamic pricing by scalable energy management systems ; field experiences and simulation results using powermatcher," in Power and Energy Society General Meeting, 2012 IEEE, july 2012, pp. 1 -8.

[14] R. Ambrosio, N. Halim, Z. Liu, D. Pendarakis, and M. G. Yao, "Method and system for efficient energy distribution in electrical grids using sensor and actuator networks," Patent US 20090228324 , March 04, 2008.

[15] K. Kok, M. J. J. Scheepers, and I. G. Kamphuis, "Intelligence in electricity networks for embedding renewables and distributed generation," ser. Intelligent Systems, Control and Automation: Science and Engineering, 2010, vol. 42.

[16] K. De Craemer, S. Vandael, B. Claessens, and G. Deconinck, "An eventdriven dual coordination mechanism for demand side management of phevs," IEEE Trans. Smart Grid, vol. PP, no. 99, pp. 1-10, 2013.

[17] F. Bliek, A. van den Noort, B. Roossien, R. Kamphuis, J. de Wit, J. van Der Velde, and M. Eijgelaar, "Powermatching city, a living lab smart grid demonstration," in Innovative Smart Grid Technologies Conf. Europe, Gothenburg. IEEE, 2010, pp. 1-8.

[18] P. Samadi, H. Mohsenian-Rad, R. Schober, and V. Wong, "Advanced demand side management for the future smart grid using mechanism design," IEEE Trans. Smart Grid, vol. 3, no. 3, pp. 1170-1180, 2012.

[19] F. Ruelens, S. Vandael, W. Leterme, B. Claessens, M. Hommelberg, T. Holvoet, and R. Belmans, "Demand side management of electric vehicles with uncertainty on arrival and departure times," in Innovative Smart Grid Technologies Conf. Europe, Berlin, 2012, pp. 1-8.

[20] B. Claessens, S. Vandael, F. Ruelens, and M. Hommelberg, "Selflearning demand side management for a heterogeneous cluster of devices with binary control actions," in Innovative Smart Grid Technologies Conf. Europe, Berlin, 2012, pp. 1-8.

[21] P. Samadi, A. Mohsenian-Rad, R. Schober, V. W. S. Wong, and J. Jatskevich, "Optimal real-time pricing algorithm based on utility maximization for smart grid," in Proc. 1st IEEE Int. Smart Grid Communications Conf., 2010, pp. 415-420.

[22] P. Tarasak, "Optimal real-time pricing under load uncertainty based on utility maximization for smart grid," in Proc. IEEE Int. Smart Grid Communications Conf., 2011, pp. 321-326.

[23] M. D. Galus, R. Waraich, F. Noembrini, K. Steurs, G. Georges, K. Boulouchos, K. Axhausen, and G. Andersson, "Integrating power systems, transport systems and vehicle technology for electric mobility impact assessment and efficient control," IEEE Trans. Smart Grid, vol. 3, no. 2, pp. 934-949, 2012.

[24] K. De Craemer and G. Deconinck, "Balancing trade-offs in coordinated phev charging with continuous market-based control," Innovative Smart Grid Technologies Conf. Europe, Berlin, 2012.

[25] M. Fahrioglu and F. Alvarado, "Using utility information to calibrate customer demand management behavior models," IEEE Trans. Power Syst.,, vol. 16, no. 2, pp. 317-322, 2001.

[26] S. Boyd and L. Vandenberghe, Convex Optimization. Cambridge University Press, Mar. 2004.

[27] D. P. Bertsekas and D. P. Bertsekas, Nonlinear Programming, 2nd ed. Athena Scientific, Sep. 1999.

[28] J. Tant, F. Geth, D. Six, P. Tant, and J. Driesen, "Multiobjective battery storage to improve PV integration in residential distribution grids," IEEE Trans. Sust. Energy, vol. 4, no. 1, pp. 182 -191, jan. 2013.
[29] P. Richardson, D. Flynn, and A. Keane, "Optimal charging of electric vehicles in low-voltage distribution systems," IEEE Trans. Power Syst., vol. 27, no. 1, pp. 268-279, 2012.

[30] Y. He, M. Petit, and P. Dessante, "Optimization of the steady voltage profile in distribution systems by coordinating the controls of distributed generations," in Innovative Smart Grid Technologies Conf. Europe, Berlin, 2012, pp. 1-7.

[31] P. Richardson, D. Flynn, and A. Keane, "Local versus centralized charging strategies for electric vehicles in low voltage distribution systems," IEEE Trans. Smart Grid, vol. 3, no. 2, pp. 1020 -1028, june 2012.

[32] G. Valverde and T. Van Cutsem, "Model predictive control of voltages in active distribution networks," IEEE Trans. Smart Grid, vol. 4, no. 4, pp. 2152-2161, Dec 2013.

[33] S. Weckx, C. Gonzalez, J. Tant, T. De Rybel, and J. Driesen, "Parameter identification of unknown radial grids for theft detection," in Innovative Smart Grid Technologies Conf. Europe, Berlin, Oct 2012, pp. 1-6.

[34] K. Turitsyn, P. Sulc, S. Backhaus, and M. Chertkov, "Options for control of reactive power by distributed photovoltaic generators," Proc. of the IEEE, vol. 99, no. 6, pp. 1063-1073, 2011.

[35] K. De Brabandere, B. Bolsens, J. Van den Keybus, A. Woyte, J. Driesen, and R. Belmans, "A voltage and frequency droop control method for parallel inverters," IEEE Trans. Power Elec., vol. 22, no. 4, pp. 11071115, 2007.

[36] L. Degroote, B. Renders, B. Meersman, and L. Vandevelde, "Neutralpoint shifting and voltage unbalance due to single-phase dg units in low voltage distribution networks," in PowerTech, 2009 IEEE Bucharest. IEEE, 2009, pp. 1-8.

[37] I. Gurobi Optimization, "Gurobi optimizer reference manual," 2014. [Online]. Available: http://www.gurobi.com

[38] N. Gatsis and G. Giannakis, "Residential load control: Distributed scheduling and convergence with lost ami messages," IEEE Trans. Smart Grid, vol. 3, no. 2, pp. 770-786, June 2012.

[39] L. Gan, U. Topcu, and S. Low, "Optimal decentralized protocol for electric vehicle charging," IEEE Trans. Power Syst., vol. 28, no. 2, pp. 940-951, May 2013.

[40] M. Kraning, E. Chu, J. Lavaei, and S. Boyd, Dynamic Network Energy Management Via Proximal Message Passing, ser. Foundations and Trends in Optimization, 2013.

[41] D. P. Bertsekas and J. N. Tsitsiklis, Parallel and distributed computation: numerical methods. Upper Saddle River, NJ, USA: Prentice-Hall, Inc., 1989.

[42] E. Peeters, C. Develder, J. Das, J. Driesen, and R. Belmans, "Linear: towards a breakthrough of smart grids in Flanders," in Proc. 2nd Int. Conf. Innovation for Sustainable Production (i-SUP 2010), vol. 3 , Bruges, Belgium, 18-21 Apr. 2010, pp. 3-6.

[43] Kabels Voor Ondergrondse Aanleg, met Synthetische Isolatie en Versterkte Mantel (Type 1kV), NBN Std. C 33-322, 1975 Std.

[44] W. Labeeuw and G. Deconinck, "Residential electrical load model based on mixture model clustering and markov models," IEEE Trans. Ind. Inform., vol. 9, no. 3, pp. 1561-1569, 2013.

[45] J. Van Roy, N. Leemput, S. De Breucker, F. Geth, P. Tant, and J. Driesen, "An Availability Analysis and Energy Consumption Model for a Flemish Fleet of Electric Vehicles," in European Electric Vehicle Congr. (EEVC), Brussels, Belgium, October 26-28, 2011.

[46] C. Cheng and D. Shirmohammadi, "A three-phase power flow method for real-time distribution system analysis," IEEE Trans. Power Syst., vol. 10, no. 2, pp. $671-679$, may 1995.

\section{BIOGRAPHIES}

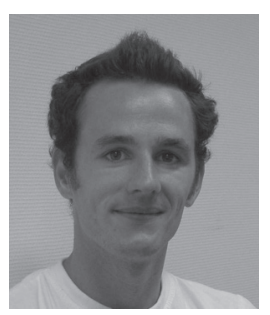

Sam Weckx (S'11) received the M.Sc. degree in Electrical Engineering in 2009 and Mechanical Engineering in 2010 from the Katholieke Universiteit Leuven (KU Leuven), Belgium, where he is currently working towards the Ph.D. degree as a research assistant with the division ESAT-ELECTA. His research interests include the application of distributed optimization in smart grids and voltage control in distribution networks. 


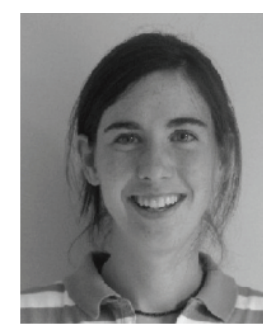

Management.

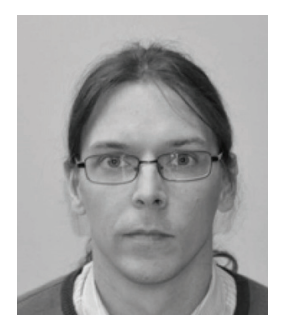

Bert Claessens received his M.Sc. and Ph.D. degrees in applied physics from the University of Technology of Eindhoven, The Netherlands, in 2002 and 2006, respectively. In 2006 he started working at ASML Veldhoven, The Netherlands, as a design engineer. Since June 2010 he has been working as a Researcher at the Vlaamse Instelling voor Technologisch Onderzoek (VITO), Mol, Belgium. His research interests include algorithm development and data analysis.

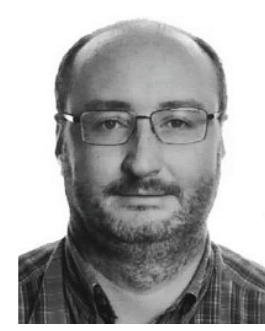

Johan Driesen (S'93 - M'97 - SM'12) received the M.Sc. and Ph.D. degrees in Electrical Engineering from the Katholieke Universiteit Leuven (KU Leuven), Leuven, Belgium, in 1996 and 2000, respectively. Currently, he is a Professor with the KU Leuven and teaches power electronics and electric drives. In 2000, he was with the Imperial College of Science, Technology and Medicine, London, U.K. In 2002, he was with the University of California, Berkeley. Currently, he conducts research on applications distributed generation, power electronics, and its 